\title{
Age-Dependent Failure of Axon Regeneration in Organotypic Culture of Gerbil Auditory Midbrain
}

\author{
Aziz Hafidi, ${ }^{1,3}$ Guo Lanjun, ${ }^{1}$ Dan H. Sanes ${ }^{1,2}$ \\ ${ }^{1}$ Center for Neural Science, New York University, 4 Washington Place, New York, New York 10003 \\ ${ }^{2}$ Department of Biology, New York University, New York, New York 10003 \\ ${ }^{3}$ Departement de Biologie, Université Blaise-Pascal, and INSERM U384, Aubière, 63170, France
}

Received 18 August 1998; accepted 8 April 1999

\begin{abstract}
Inferior colliculus (IC) slice cultures from postnatal (P) day 6-8 gerbils exhibit axonal regeneration across a lesion site, and these regrowing processes can form synapses. To determine whether regenerative capacity is lost in older tissue, as occurs in vivo, slices from P12-21-day animals were grown under similar conditions. While these cultures displayed a near complete loss of neurons over 6 days in vitro, glutamate receptor antagonists (AP5 and/or CNQX) significantly enhanced survival, particularly at P12-15. In contrast, several growth factors or high potassium did not improve neuron survival. Therefore, axonal regeneration was assessed following complete transection of the commissure in AP5/CNQX-treated IC cultures from P12 animals. Neurofilament staining revealed that transected commissural axons survived for 6 days in vitro, but only a few processes crossed the lesion site and these axons did not extend into the contralateral lobe. In
\end{abstract}

contrast, there was robust axonal sprouting and growth within one lobe of the IC, remote from the lesion site. When P6 and P12 tissue was explanted onto a coated substrate, the P6 axons grew onto the substrate, but the $P 12$ axons were seemingly prevented from reaching the substrate by a veil of nonneuronal cells. Coculture of the IC and one of its afferent populations, the lateral superior olive, provided a similar finding, indicating that failure to regenerate was a general property at the age examined. These data show that neuron survival is not sufficient to permit axon regeneration at $\mathbf{P 1 2}$, and suggest that P12 lesion sites manufacture a prohibitive substrate since process outgrowth is blocked specifically at the commissure transection. () 1999 John Wiley \& Sons, Inc. J Neurobiol 41: 267-280, 1999

Keywords: regeneration; cell death; glutamate receptors; inferior colliculus
The neonatal mammalian central nervous system retains the capacity to regenerate central axonal processes following injury, but this property is lost during early development (Cajal, 1928; Kalil and Reh, 1982; Fawcett et al., 1989; Shimizu et al., 1990; Li et al., 1995; MacLaren and Taylor, 1995; Wang et al., 1996). Even axons that are able to regenerate usually grow around the lesion site (Kalil and Reh, 1982; Bregman and Goldberger, 1983; Xu and Martin,

Correspondence to: D. H. Sanes

Contract grant sponsor: NIH; contract grant number: DC00540

(C) 1999 John Wiley \& Sons, Inc. CCC 0022-3034/99/020267-14
1989; Hasan et al., 1993). The failure of adult axons to regenerate may be due to the absence of growth permissive signals or the presence of growth inhibitory cues at the site of injury (Carbonetto, 1991).

The importance of extracellular cues in vivo is clearly illustrated by the ability of peripheral nerve grafts or glia ensheathing cells to support central axonal regrowth (Richardson et al., 1980; David and Aguayo, 1981; Aguayo et al., 1990; Li et al., 1997; Ramon-Cueto et al., 1998), the growth inhibitory effect of myelin-related proteins (Keirstead et al., 1992; Bandtlow et al., 1990; MacKerracher et al., 1994; Mukhopadhyay et al., 1994), and growth inhibition by 
scar tissue (Reier et al., 1983; Rudge and Silver, 1990; Davies et al., 1997). However, it is also the case the mature axons have are more limited in their capacity to grow in a given environment (Fawcett, 1992; Chen et al., 1995; Li et al., 1995).

It is also likely that axonal regeneration is precluded by atrophy and cell death. For example, axotomized neurons may be deprived of a trophic interaction with their target. Disruption of calcium homeostasis by improper levels of glutamatergic transmission may also result in cell death. Thus, glutamate receptor antagonists are often found to promote neuron survival under several experimental conditions (Choi et al., 1988; Michaels and Rothman, 1990; Agnew et al., 1993; Pozzo-Miller et al., 1994; Solum et al., 1997).

We previously demonstrated that inferior colliculus (IC) slices from postnatal day (P) 6-8 gerbils can be maintained in organotypic culture for up to 2 weeks, and that IC neurons can regenerate their axons through a total lesion of the commissural pathway. Furthermore, the regenerated axons are able to form synaptic contacts in the contralateral IC (Hafidi et al., 1995). The gerbil has been used in these studies because we are interested in functional recovery and we have previously described the in vivo and in vitro physiology in this species (Sanes and Wooten, 1987; Hafidi et al., 1996; Lo et al., 1998; Moore et al., 1998; Sanes et al., 1998; Thornton et al., 1999; Vale and Sanes, 1999). When we attempted to study the regenerative capacity of axons in animals at P12 and older, there was a complete absence of neuron survival in IC organotypic cultures. Therefore, we are interested in determining whether neuron survival is a sufficient precondition to permit axon regeneration in this system. Toward this end, growth factors and glutamate receptor antagonists were employed in an attempt to promote survival, and the regenerative capacity of the rescued neurons was assessed in P12 cultures.

\section{MATERIALS AND METHODS}

\section{Organotypic Cultures}

Gerbils (Meriones unguiculatus) at P12-21 were used for organotypic culture experiments, as described previously (Stoppini et al., 1991; Hafidi et al., 1995). Animals were alcohol-sterilized and decapitated, and the brains were removed and put in a cooled solution of minimum essential medium (MEM) containing $0.6 \mathrm{~g} / \mathrm{mL}$ of glucose. Vibratome sections of $250 \mu \mathrm{m}$ were collected in MEM at $4^{\circ} \mathrm{C}$, wellpreserved slices were selected, and the pial membrane was removed. Sections were transferred through several drops of the culture medium (50\% MEM, 25\% Earle's balanced salts, $25 \%$ horse serum; Gibco-BRL), and placed on $0.2-\mu \mathrm{m}$ filter inserts (Millipore) within the wells of a 24-chamber plate (Falcon). The culture medium was changed at 1- to 2-day intervals.

As shown in Figure 1, four types of cultures were used in this study: (a) bilateral IC slices with an intact commissural projection ( $n=13$ slices at $\mathrm{P} 12, n=4$ slices at $\mathrm{P} 21)$; (b) bilateral IC slices in which the entire commissure was severed at the midline, resulting in two pieces that were explanted side by side $(n=35$ slices at P12, $n=3$ slices at P15, $n=3$ slices at P21); (c) unilateral IC and unilateral superior olivary complex (SOC) slices (Sanes and Hafidi, 1996) at the level of the lateral superior olivary nucleus (LSO), explanted side by side with the ventral IC in contact with the dorsal SOC ( $n=17$ slices at P12); and (d) unilateral IC central nucleus slice $(n=6$ slice at P6, $n$ $=16$ slices at P12) plated on a filter insert coated with polyornithine and laminin (Sigma).

Growth factors were added to the N2 defined medium or serum-containing medium at $50 \mathrm{n} M$ for brain-derived neurotrophic factor (BDNF) or neurotrophin-3 (NT-3) (Regeneron), $100 \mathrm{n} M$ for nerve growth factor (NGF; Gibco), and $50 \mathrm{n} M$ for fibroblast growth factor (FGF; Gibco). In some experiments, BDNF and NT-3 were added together. High potassium levels were obtained by adding potassium chloride $(\mathrm{KCl})$ to the serum-containing medium at a final concentration of 15-75 $\mathrm{mM}$. The growth media was supplemented with the ionotropic glutamate receptor antagonists, 6-cyano-7-nitroquinoxaline-2,3-dione (CNQX; $100 \mu M$; RBI) and D,L-2-amino-5-phosphonovaleric acid (AP5; 100 $\mu M$; RBI), as indicated in the Results.

After 6 days in vitro (DIV), slices were immersion fixed in $4 \%$ paraformaldehyde in $0.12 M$ phosphate buffered saline (PBS) for $1 \mathrm{~h}$, rinsed in PBS, and then incubated in $1 \% \mathrm{H}_{2} \mathrm{O}_{2}$. After several washes in PBS, the free-floating sections were incubated in 5\% goat serum for $30 \mathrm{~min}$ and transferred to one of four primary antibodies (mouse anti$\beta$-tubulin, provided by Dr. A. Frankfurter, University of Virginia, Charlottesville, VA; mouse anti-MAG, provided by Dr. M. Schachner, Swiss Federal Institute of Technology, Zurich, Switzerland; mouse anti-neurofilament, provided by Dr. D. Dahl, Boston, Massachusetts; and mouse anti-GAP43, Sigma Chemical) overnight at $4^{\circ} \mathrm{C}$. Slices were washed in several changes of PBS, and then incubated in the appropriate secondary antibody (biotinylated goat anti-rabbit or goat anti-mouse; Sigma). Sections were again washed in PBS and then incubated in avidin-biotin-horseradish peroxidase complex (ABC-HRP; Vector Labs) for $2 \mathrm{~h}$. The tissue was washed in $0.1 M$ Tris-buffered solution (TBS), incubated in diaminobenzidine (DAB; $50 \mathrm{mg} / 100$ $\mathrm{mL}$ TBS) for $15 \mathrm{~min}$, and then reacted in a DAB solution with $0.03 \% \mathrm{H}_{2} \mathrm{O}_{2}$. Sections were mounted on gelatin-coated slides, dehydrated, and coverslipped with permount.

\section{Semithin Sections}

After 6 DIV, slices were fixed in $4 \%$ paraformaldehyde and $2 \%$ glutaraldehyde in $0.1 \mathrm{M}$ phosphate buffer. The slices 
A

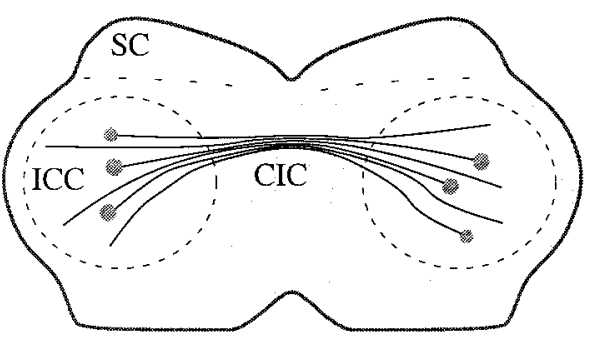

\section{B Commissure Transection (P12)}

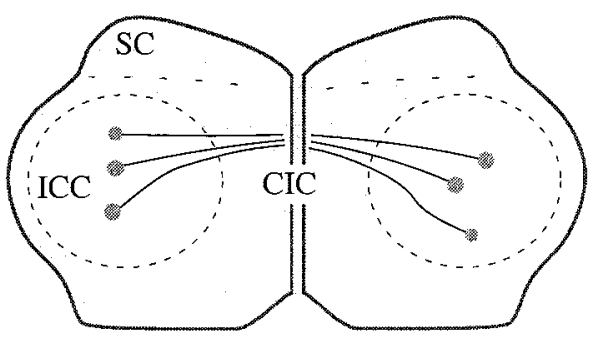

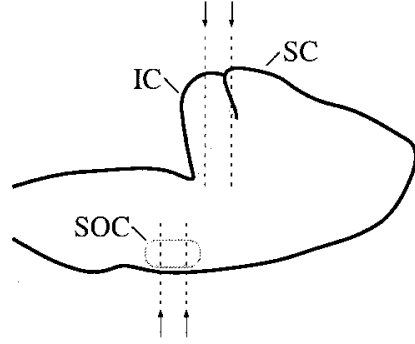

$\mathrm{D}$ $\underline{\text { ICC on Substrate (P6, P12) }}$

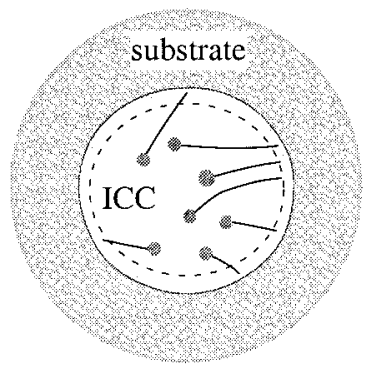

Figure 1 Schematic of the four culture conditions employed in this study. (A) Transverse sections of the IC from P12 animals containing an intact commissure were grown for 6 DIV. (B) The commisure was transected in transverse IC sections from P12 animals, and the sections realigned and grown for 6 DIV. (C) Transverse sections of one IC lobe and the superior olivary nuclei from P12 animals were aligned as shown, and grown for 6 DIV. (D) The central nucleus of IC from P6-21 animals was placed on substrate and grown for 6 DIV. ICC-central nucleus of the inferior colliculus; SC-superior colliculus; CIC-commissure of the inferior colliculus; substrate, polyornithine-, and laminin-coated insert.

were washed, osmificated in $1 \% \mathrm{OsO}_{4}$, incubated in uranyl acetate, dehydrated, and embedded in epoxy resin. Semithin sections $(1 \mu \mathrm{m})$ were obtained on an LKB ultramicrotome, stained with $0.5-1 \%$ Toluidine blue in saturated borate, and mounted for light microscopic observation.

\section{Morphological Criteria for Cell Counts}

The morphological criteria used to count neurons are identical to those described previously (Hafidi et al., 1994). Only clearly recognizable neurons (large soma, substantial cytoplasm, and large nucleus with nucleolus) were counted.
However, one limitation of the counting method is that abnormally small neuronal somata would not have been included in these counts. Neurons that contain vacuoles in their cytoplasm were not counted. Astrocytes were distinguished by a small cell body, lightly stained cytoplasm, and lightly stained nucleus with a thin rim of condensed chromatin lining the membrane. Oligodendrocytes were distinguished by dense cytoplasm and nucleoplasm (clumps of chromatin). All counts were taken from the center of the explant in an $80-\mu \mathrm{m}^{2}$ grid using a $\times 100$ oil objective. Three squares were counted in each section, and sampled sections were a minimum of $10 \mu \mathrm{m}$ apart. Control counts were 

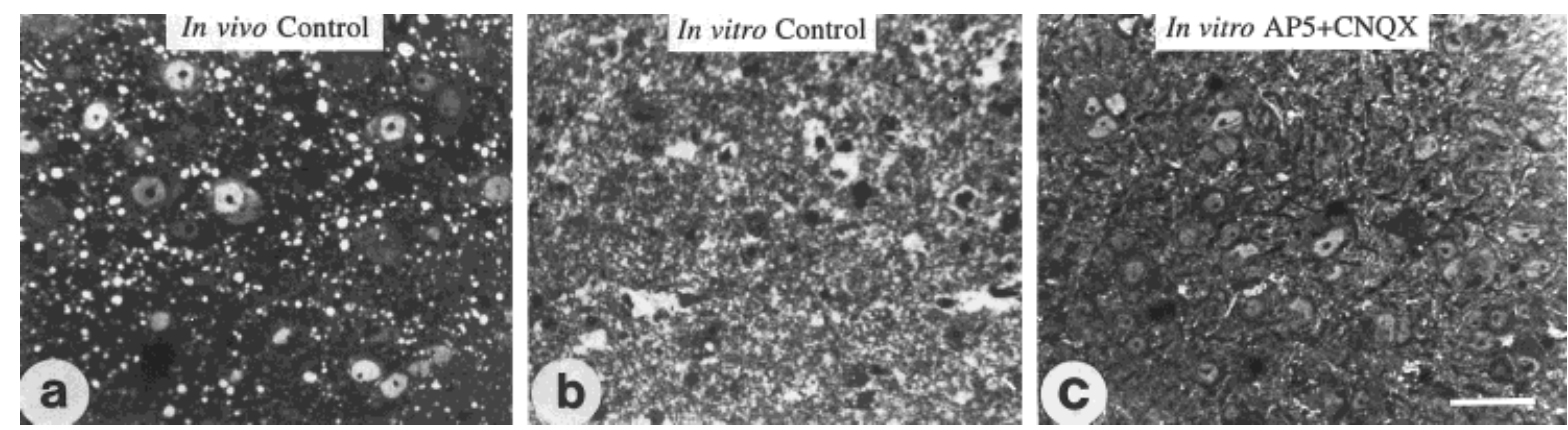

Figure 2 Neuron survival under different conditions is shown in semithin sections. (A) An in vivo P12 slice did not display dying neurons. (B) A control P12 organotypic culture displayed many dying neurons after 6 DIV. (C) A P12 slice grown for 6 DIV in glutamate receptor antagonists (AP5 and CNQX). Neurons and glia are present within the slice at a level far above that seen in control media, and these neurons exhibited well-preserved morphologies. Bar $=40 \mu \mathrm{m}$.

obtained from P12 in situ tissue that was prepared in an identical manner to the cultured tissue (see above), and organotypic tissue that was incubated for 6 DIV in serumcontaining medium or $\mathrm{N} 2$ defined medium without the addition of growth factors, $\mathrm{KCl}$, or glutamate receptor antagonists.

\section{RESULTS}

\section{Glutamate Receptor Antagonists and Neuron Survival}

Control tissue obtained from freshly cut slices that were fixed immediately exhibited well-preserved neuron morphology. Neuronal perikarya did not display any characteristic of cell death beneath the vibratomecut surface [Fig. 2(A)]. Neurons did not contain vacuoles in their cytoplasm, although some cells had darkly stained cytoplasm. The extracellular space was prominent, as expected in tissue of this age. In contrast, IC slices that were grown in normal or defined N2 media for 6 DIV exhibited a dramatic loss of neurons. In many sections there was a total absence of neuronal cell bodies, particularly within the center of the explant [Fig. 2(B)]. A few glial cell bodies, however, were present in the explants.

Brain-derived neurotrophic factor and NT3 were added, alone or in combination, to cultures from P15 animals, an age at which neurotrophin receptors are highly expressed in the gerbil IC (Hafidi et al., 1996). Semithin sections from the neurotrophin-treated slices did not reveal a positive action on neuron survival as compared to the in vitro control slices (not shown), beyond a very few remaining neurons at the edge of the explants. Most of the cells present in the explant appeared to be phagocytic glial cells with microglialike features (cf. small cell bodies and nuclei, and very dense nucleoplasm with patches of chromatin). Similarly, NGF did not enhance neuronal survival, nor did FGF (not shown). Although depolarizing agents promote neuron survival in vitro at young ages (Franklin et al., 1995; Lohmann et al., 1998), increasing the $\mathrm{KCl}$ concentration $(25,50$, or $75 \mathrm{mM})$ in the serum-containing culture medium did not enhance IC neuron survival, although there were a large number of cells with astrocytic features.

The ameliorative effect of glutamate antagonist treatment on IC neuron survival was pronounced [Fig. 2(C) and Table 1]. Slices treated with AP5 or CNQX, or both in combination, contained neurons with a preserved morphology across the entire explant. Three different neuron morphologies were observed in semithin sections from treated cultures. One population of neurons appeared healthy, without the presence of cytoplasmic vacuoles or other signs of structural degeneration. There was no differences between

Table 1 Neuron Survival Improved by Glutamate Receptor Antagonists Compared to Control In Vivo and In Vitro Conditions

\begin{tabular}{lcllcr}
\hline & AP5 + CNQX & AP5 & CNQX & In Vitro Control & In Vivo Control \\
\hline Neurons $/ 80 \mu \mathrm{m}^{2}$ & $8.9 \pm 1.7$ & $4.2 \pm 1.2$ & $3.5 \pm 0.7$ & 0 & $6.4 \pm 1.1$ \\
Total neurons & 145 & 97 & 67.9 & & 115 \\
No. of slices & 19 & 23 & 20 & 8 & 20 \\
\hline
\end{tabular}


this morphology and that found in freshly cut control tissue from P12 animals. A second population of neurons appeared pyknotic and contained cytoplasmic vacuoles. The number of vacuoles per neuron was variable but tended to be greatest when the neuron showed other signs of cell death (cf. eccentric nucleus, shrunk and darkly stained nucleus, darkly stained cytoplasm). These first two subpopulations represent the majority of neurons observed in treated cultures. A third, infrequent group of neurons (2-3/ section) exhibited a healthy morphology except for dense chromatin clumping, sometimes with a rosettelike appearance, inside the nucleus.

Only the neuronal population that exhibited a healthy morphology (the first subtype described above) was counted, and the results are presented in Table 1. The cell counts showed that either AP5 or CNQX alone was able to rescue a significant number of neurons, although AP5 had a more potent affect. When used in combination, AP5 and CNQX had a greater ameliorative affect than either alone. In P15 and P18 explants, both neurons and glial cells could occasionally be found after 6 DIV, although the number of pyknotic neurons clearly increased with age of explanation. Dying neurons were rarely observed in P21 cultures, suggesting that cell death occurred rapidly in these cultures. A very few healthy neurons were present in P21 cultures after 6 DIV, although the explants were filled with morphologically preserved astrocytes and oligodendrocytes.

\section{Axonal Regeneration}

Having identified a procedure (AP5 and CNQX) that maintained P12 IC slices in vitro for 6 DIV, we tested whether transected commissural axons were able to cross the lesion site, as occurs in P7 cultures (Hafidi et al., 1995). The axons were stained with an antibody directed against neurofilament (NF), and staining was occasionally present in neuronal cell bodies. In P12 slices with an intact commissure, the NF antibody apparently stained most axons within both lobes of the IC, and the commissural axons that coursed through the midline [Fig. 3(A,B)]. In P12 slices with a transected commissure, robust NF staining was observed in axons within each central nucleus of the IC, as well as the cut commissural axons that remained at the midline [Fig. 3(C-H)]. Many labeled commissural axons terminated in large varicosities or terminal bulbs (Fig. 4). The vast majority of these commissural axons did not cross the lesion site, and a total of 15 crossing axons were observed ( $n=23$ cultures). However, these axons did not extend for a long distance and never traversed the entire lesion site to reach the contralateral IC nucleus. A few axons also ran along the lesion without penetrating and crossing it [Fig. 3(F,H)], and a few were observed to grow back into the ipsilateral IC.

MAG immunostaining revealed both oligodendrocyte cell bodies and myelinated fascicles throughout P12 IC cultures. In P12 slices with a transected commissure, MAG-positive fibers could be found within the central nucleus and around its edge, but no staining was observed crossing the midline lesion (Fig. 5).

\section{Axon Regeneration in Cocultures}

To test whether a different axonal projection to the IC was better able to regenerate across a lesion, a region of P12 SOC containing the lateral superior olivary nucleus (LSO) and the medial nucleus of the trapezoid body (MNTB) was cocultured with one lobe of the IC for 6 DIV. Robust NF immunoreactivity was observed in axons and some neuronal cell bodies on both sides of the explant. At the site of contact (dorsal SOC placed adjacent to ventral IC), a larger number of axons were observed to cross the lesion (Fig. 6) compared to the transected IC slices. The number of crossing axons in the SOC-IC slices varied from 0 to 36 axons per slice and there were a total of 166 crossing axons ( $n=17$ cultures). In general, the regenerating axons were very large caliber and some of them emerged from the reticular formation. GAP43-stained sections also displayed a number of axons that crossed the lesion (not shown), and these also tended to be large caliber axons.

\section{Axon Outgrowth on Substrate-Coated Inserts}

To determine the growth potential of P6 and P12 axons, inserts were coated with a mixture of polyornithine and laminin, and the outgrowth of axons from the central nucleus of the IC was assessed in both N2-defined and serum-containing medium. Slices from P12 animals were grown in the presence of glutamate receptor antagonists, but slices from P6 animals were not. In defined medium, robust axonal outgrowth onto the substrate was observed in P6 explants [Fig. 7(A,B)], while the outgrowth in P12 explants was quite limited [Fig. 7(C,D)]. In serumcontaining medium, both P6 and P12 explants exhibited very little axonal outgrowth onto the substrate [Fig. 7(E-H)]. A perimeter of nonneuronal cells surrounded the explant shown in Figure $7(G)$, and the stained axons grew within the explant but did not extend onto this veil. 
A

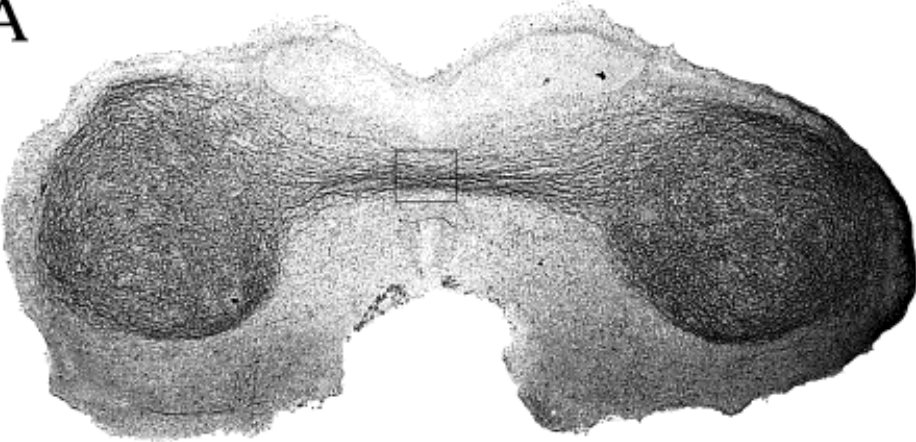

$\mathrm{C}$

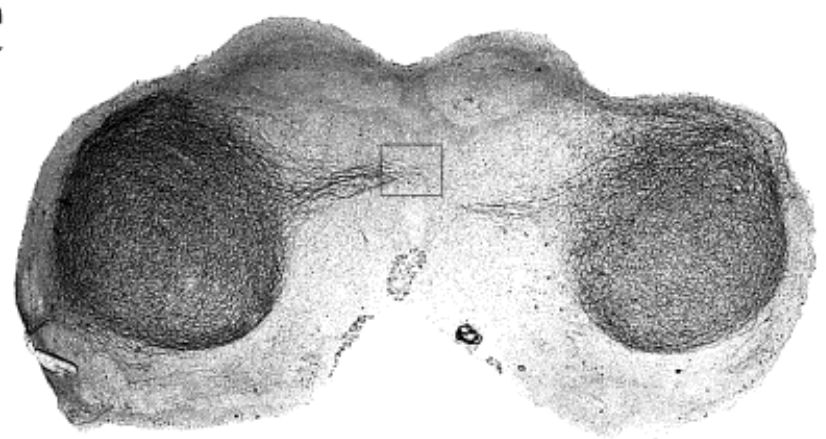

$\mathrm{E}$

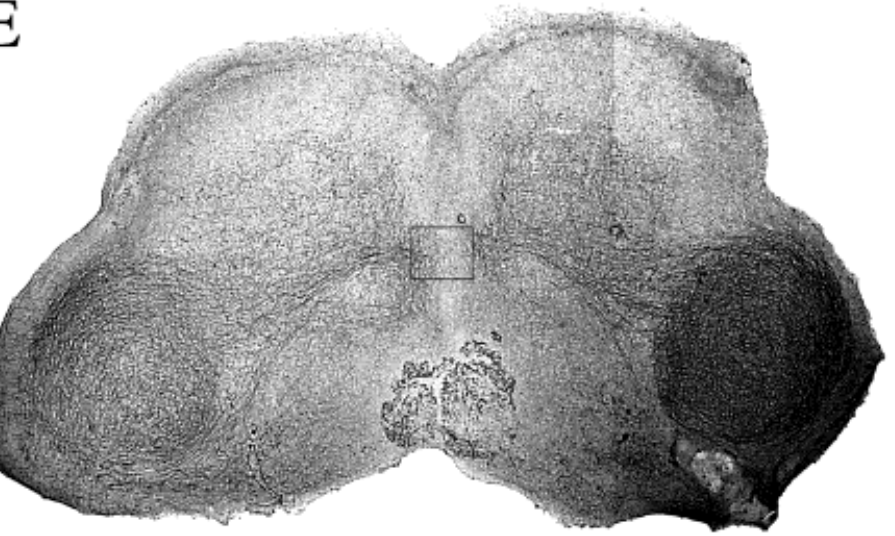

G

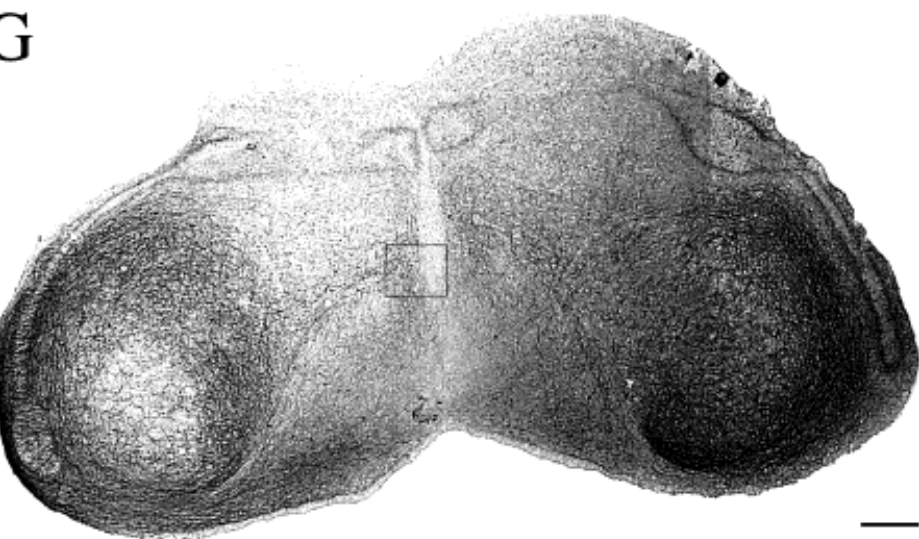

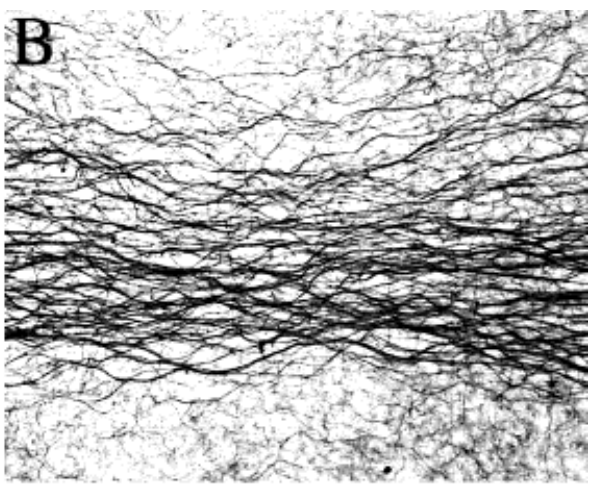
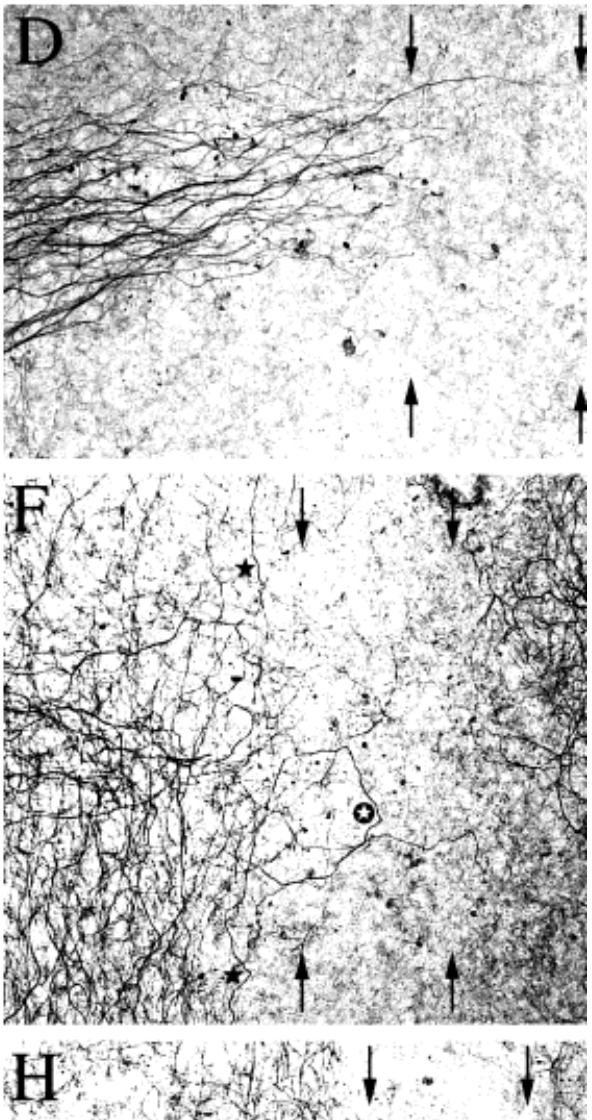

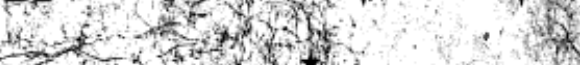

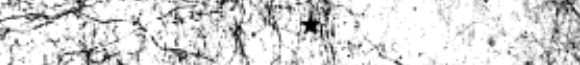

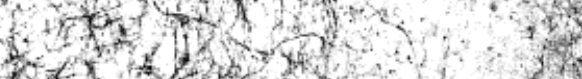

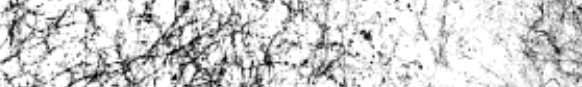

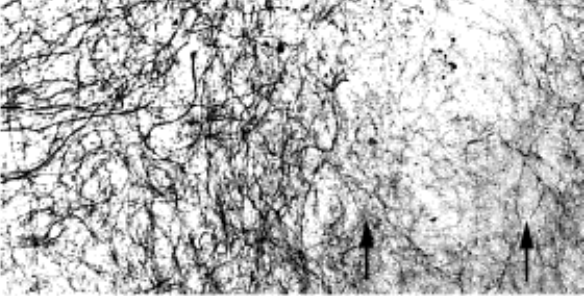

Figure 3 Neurofilament (NF) staining of P12 IC slices grown from 6 DIV with intact or transected commissure. (A,B) In a control section, NF labeling is present in axons throughout both sites of the slice as well as axons coursing through the midline. (B) At higher magnification [box in (A)], a 

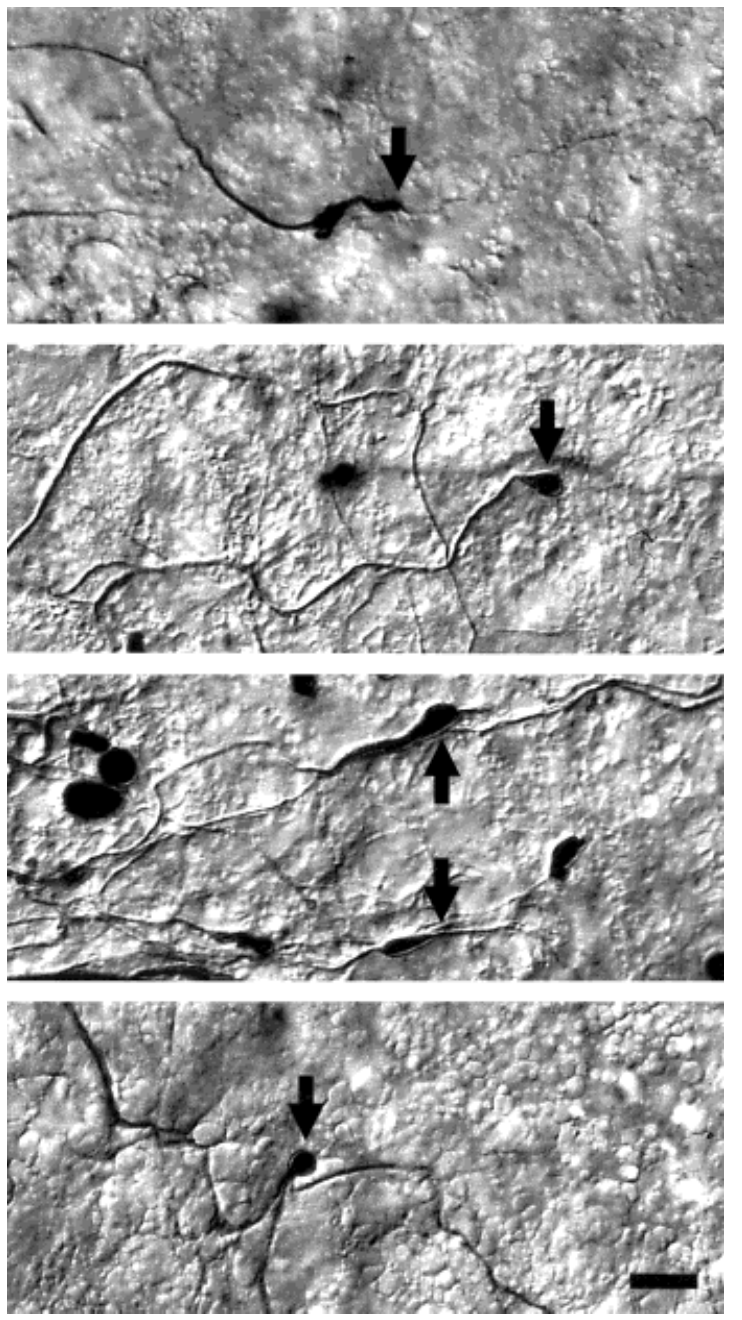

Figure 4 Four examples of the enlarged terminal endings (arrows) of cut axons near the midline. Bar $=10 \mu \mathrm{m}$.

\section{DISCUSSION}

In a previous study, we reported that cut axons would regenerate across the lesion site in $\mathrm{P} 7$ organotypic cultures of auditory midbrain (Hafidi et al., 1995). The present study examined the regenerative capacity of central auditory axons from juvenile animals at P12. At first, IC neurons from $\geq \mathrm{P} 12$ animals did not survive in the organotypic conditions that had previously proved suitable for P7 tissue. However, it was possible to rescue a significant fraction of P12 neurons with the addition of the glutamate receptor antagonists, AP5 and CNQX (Fig. 2 and Table 1). Most importantly, the prevention of cell death allowed axons to survive within the explant, thus making it possible to study regeneration in vitro. The results indicate that neuron survival was not sufficient to permit axon regeneration across a lesion site. Furthermore, failure to regenerate was apparently due to a nonpermissive environment at the lesioned midline.

\section{Cell Survival and Glutamate Receptor Antagonists}

The data reveal a nearly complete loss of neurons in IC tissue explanted from P12 and older animals. This cell death appears to be glutamate associated. Neither supplementing the medium with growth factors nor continuous depolarization with elevated potassium had a positive effect on neuronal survival. In contrast, addition of ionotropic glutamate receptor antagonists rescued a significant number of neurons in P12 explants (Table 1). Either AP5 or CNQX alone prevented many neurons from degenerating, but AP5 appeared to have a more potent survival action. Moreover, the two antagonists seemed to have a synergistic action (Table 1), as has been found in hippocampal cultures in which glutamate levels are raised with a transport blocker (Vornov et al., 1995). The higher neuron counts observed in the AP5/CNQX-treated slices compared to freshly cut control tissue were probably due to the gradual thinning of organotypic slices from an initial thickness of $300 \mu \mathrm{m}$ to a final thickness of $100 \mu \mathrm{m}$. These results suggest that excitotoxic injury to IC neurons occurs during tissue preparation at P12. In contrast, P6 neurons survive without

dense array of NF-positive axons were observed crossing the midline. (C-H) In P12 slices grown for 6 DIV with a transected commissure, NF staining was present in axons throughout the IC central nucleus of each side, and commissural axons survived at the lesion site. As shown at higher magnification (D,F,H), these axons did not cross the lesion site, although some of them did grow along it or back into the ipsilateral IC [see stars in $(\mathrm{F}, \mathrm{H})]$. Some cut endings terminated as large varicosities or terminal bulbs. A very few axons grew into the lesion site but did not traverse the full distance to reach the other IC lobe (arrows). Sections shown in (A-C) were grown in the presence of AP5 and CNQX for 6 DIV, and the section in (D) was grown in AP5 and CNQX for 3 DIV. Bar $=300 \mu \mathrm{m}$ for low-power micrographs (left column) and $40 \mu \mathrm{m}$ for high-power micrographs (right column). 

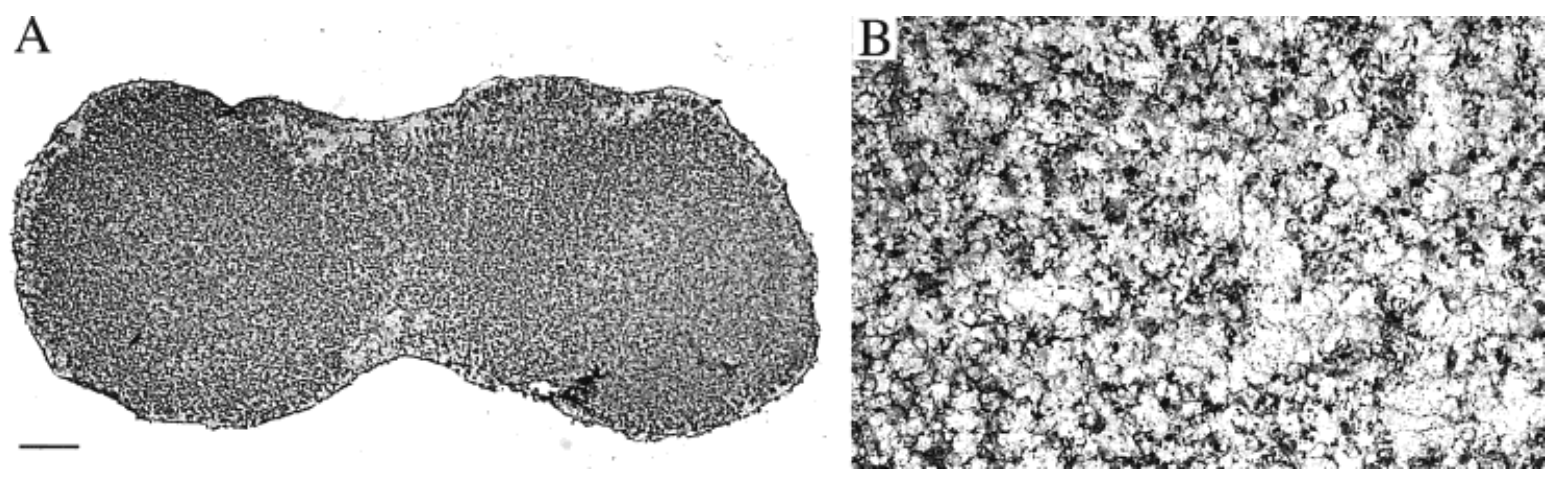

Figure 5 MAG staining in P12 organotypic slice at 6 DIV following transection of the commissure. (A) MAG-positive oligodendrocytes and axonal sheaths were found within both side of the slice. (B) At high magnification, MAG-positive fibers were not found at the midline. Bar $=300 \mu \mathrm{m}$ for (A) and $40 \mu \mathrm{m}$ for (B).

the addition of glutamate receptor blockers (Hafidi et al., 1995).

The present data are in agreement with previous reports of rat P15 cerebellar organotypic cultures that show a total loss of Purkinje neurons (Dusart et al., 1997), and from hippocampus where spontaneous pyramidal cell death was prevented by the block of glutamate ionotropic receptors (Pozzo-Miller et al., 1994). The latter study indicates that glutamate receptors mediate decreased survival of CNS cultures from P12 and older tissue. The vulnerability of neurons to glutamate during this developmental period may be due to the acquisition of specific glutamate receptor subclasses (Watanabe et al., 1994). The degree of IC maturation is clearly a key factor in glutamate cytotoxicity in vitro, since slices from P6 animals survive in normal serum-containing media (Hafidi et al., 1995).

Activation of glutamate receptors is thought to result in neuronal injury and death by increasing intracellular free calcium. A number of previous studies have shown that neuron can be protected against such excitotoxicity by preventing the elevation of calcium using glutamate receptor antagonists or calcium channel blockers (Choi and Hartley, 1993) or by buffering intracellular calcium (Tymianski et al., 1994). AMPA/kainate receptor-mediated cytotoxicity depends on extracellular calcium (Hori et al., 1985; Rothman et al., 1987; Ellren and Lehmann, 1989; Brorson et al., 1994; Lu et al., 1996), and the $N$ methyl-D-aspartate (NMDA) receptor-coupled channel is known to have a significant permeability to calcium (MacDermott et al., 1986). Conditions that limit influx of calcium through NMDA receptors are particularly efficient in blocking glutamate excitotoxicity.

An in situ hybridization study revealed an expres- sion of NMDA receptors (R1 and R2A subunits) in the rat inferior colliculus that increases during the second postnatal week (Watanabe et al., 1994). This NMDAR expression may correlate with the increased vulnerability of IC neurons to the endogenous glutamate in our P12 explants. Nevertheless, the ameliorative effect of glutamate receptor antagonists declined in older tissue.

Depolarizing agents such as high $\mathrm{KCl}$ have been used successfully to promote neuronal survival in vitro (Franklin et al., 1995; Lohmann et al., 1998). However, increasing the potassium concentration in our culture medium did not have a positive effect on neuron survival. This was expected, since membrane depolarization would likely increase calcium influx through voltage-gated channels (Kingsbury and Balazs, 1987) or glutamate release (Didier et al., 1993). However, it is important to note that a recent study has shown depolarization to be crucial to the survival of rat auditory brainstem explants obtained from early postnatal animals (Lohmann et al., 1997).

The presence of the neurotrophin receptors in the developing IC (Hafidi et al., 1996) suggested that the addition of specific growth factors to the culture media might enhance neuron survival. However, neither the use of three neurotrophins nor FGF rescued neurons in P12 or older cultures. It is possible that glutamate-associated cell death was too fast for the growth factors to have a beneficial affect (Mattson et al., 1993). Moreover, BDNF may actually increase the electrical activity of neurons (Shen et al., 1994), and could have potentiated cell death in our system (Koh et al., 1995). Thus, these growth factors may not constitute a survival signal for the IC neurons at this stage.

Most dying neurons within the slice cultures exhibited distinct structural characteristics, including the 

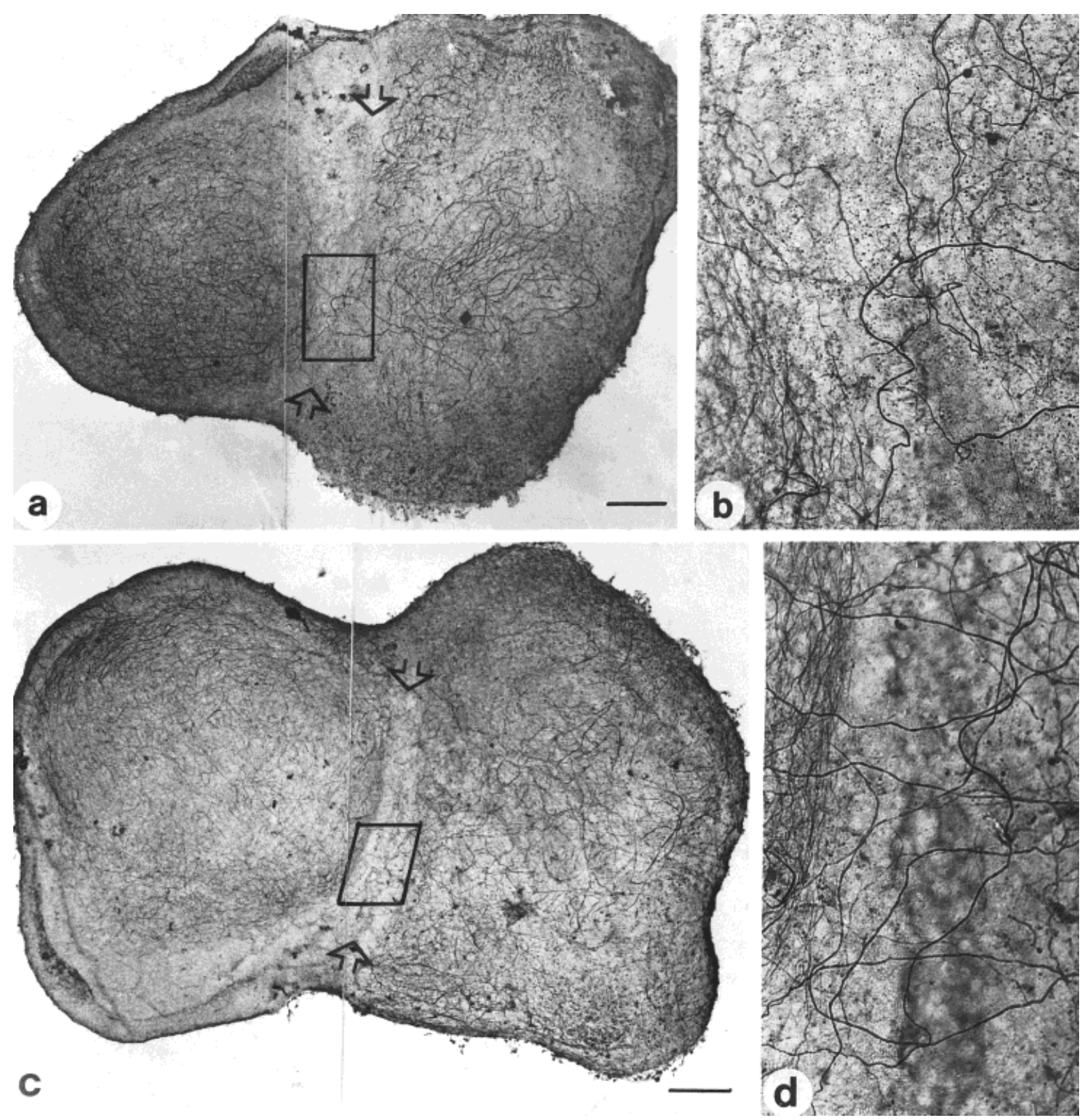

Figure 6 Two examples of neurofilament-stained cocultures of the IC and the SOC from a P12 animal grown for 6 DIV. (A) A coculture is shown at low magnification with IC to the left of the arrows, and the SOC on the right. The interface between the two slices (box) is shown at higher magnification in (B). (B) NF staining showed some fibers at the interface between the two sections, although many more avoided the lesion site. A few thick axons from the SOC slice were observed to cross the lesion site, but they may emanate from reticular neurons. Bar $=280 \mu \mathrm{m}$ for (A) and $70 \mu \mathrm{m}$ for (B). (C) Neurofilament-stained cocultures of the IC and the SOC from a P12 animal grown for 6 DIV. (C) A second coculture is shown at low magnification with IC to the left of the arrows, and the SOC on the right. The interface between the two slices (box) is shown at higher magnification in (D). (D) Many thick axons from the SOC slice were observed to cross the lesion site. Both cocultures were treated with AP5 and CNQX for 6 DIV. Bar $=260 \mu \mathrm{m}$ for (C) and 50 $\mu \mathrm{m}$ for (D).

presence of vacuoles within their cytoplasm that increased with age of explant. A few neurons had a normal morphology, except that their nuclei contained chromatin clumping. These cells may represent an early stage of the cell death process. The presence of vacuoles in neurons has been associated with injury 

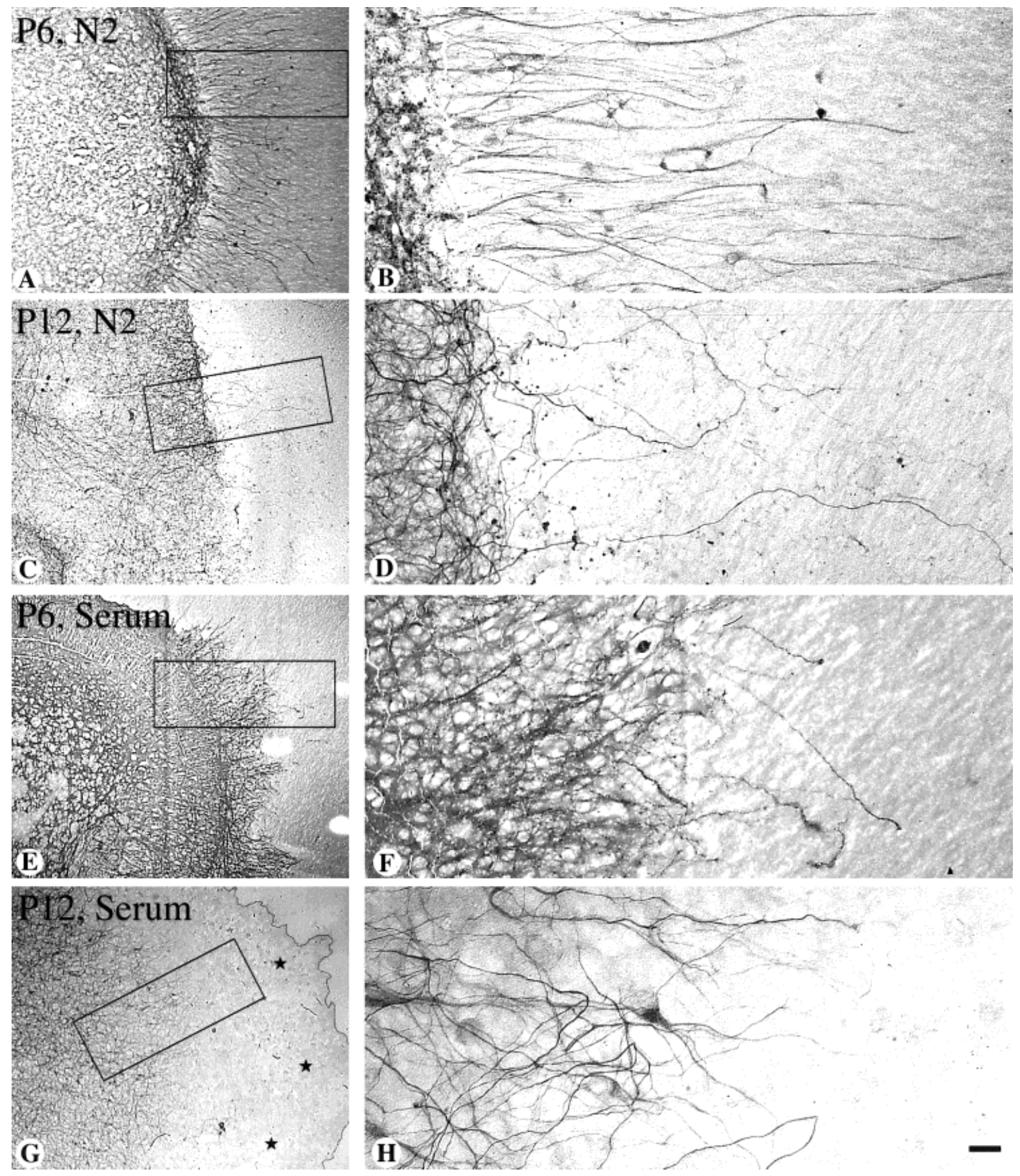

Figure 7 Explants of the central nucleus of the IC grown on polylysine and laminin coated inserts. $(A, B)$ P6 tissue grown in N2 defined medium displayed robust axonal outgrowth onto the substrate. The axons shown at higher magnification (B) are from the area delineated by the box in (A). (C,D) P12 tissue grown in N2-defined medium displayed little axonal outgrowth compared to that seen at P6. The axons shown at higher magnification (D) are from the area delineated by the box in (C). (E,F) P6 tissue grown in serum-containing medium displayed little axonal outgrowth onto the substrate. The axons shown at higher magnification $(\mathrm{F})$ are from the area delineated by the box in (E). $(\mathrm{G}, \mathrm{H}) \mathrm{P} 12$ tissue grown in serum-containing medium displayed no axonal outgrowth onto the substrate. Instead, these axons appeared to avoid the veil of glial cells (stars) that surrounded each explant (outer limit is denoted by line). The axons shown at higher magnification (D) are from the area delineated by the box in (C). The P6 cultures were stained for $\beta$-tubulin and P12 cultures were stained for neurofilament (see Materials and Methods). P12 slices were cultured in glutamate receptor antagonist-containing medium. Bar $=320 \mu \mathrm{m}$ in $(\mathrm{A}, \mathrm{C}, \mathrm{E}, \mathrm{G})$ and $40 \mu \mathrm{m}$ in $(\mathrm{B}, \mathrm{D}, \mathrm{F}, \mathrm{H})$. 
with glutamate or its agonists (Sperk et al., 1983; Clarke, 1990; Portera-Cailliau et al., 1995). The pattern of neuronal injury observed here correlates with a slow injury characterized by vacuolation and chromatin clumping. At present, we have no direct evidence concerning the mode of cell death (Wyllie et al., 1980; Van-Lookeren et al., 1995).

In P21 slices, the cell death appeared different from that observed in P12 cultures since few degenerating neuron cell bodies were observed after 6 DIV. However, swollen perikarya were observed at this age, suggesting that cell death and their clearance were occurring more rapidly in P21 tissue, and necrosis is likely to be involved.

\section{Axonal Regeneration}

In a previous study, we showed that IC cultures from an early postnatal period (P6-8) can survive in vitro and retain the capacity to regenerate axonal processes through a midline commissural lesion (Hafidi et al., 1995). The regenerated axons can make synaptic contacts within the contralateral IC neurons. After rescuing a significant fraction of neurons in P12 cultures, we examined whether they also had the ability to regenerate axonal processes. Glutamate antagonisttreated P12 cultures displayed an intact commissural projection in vitro. However, following a commissure transection, there was almost a complete absence of regeneration across the lesion (Fig. 3). There were only a few axons that crossed the lesion in P12 cultures, although there were many axonal processes on either side of the midline lesion. Therefore, the capacity to regenerate axons is present at $\mathrm{P} 12$, but the ability to traverse a lesion site seems to decrease with age. As discussed below, these results lead us to conclude that (a) the lesion site contains one or more growth inhibitory molecules, (b) P12 axons have some growth potential and would be able to extend across a lesion site in the absence of these putative signals, and (c) MAG-positive oligodendrocytes are probably not involved in this inhibition. Sprouting may be robust within each lobe of the IC both because the MAG-positive cells are immature and do not express growth-inhibitory molecules, and because there is not an injury site within the depth of the tissue.

This result is in accordance with previous studies showing a transient period for axonal outgrowth across a lesion site (Kalil and Reh, 1982; Xu and Martin, 1989; Shimizu et al., 1990; Hasan et al., 1993; Li et al., 1995; MacLaren and Taylor, 1995; Dusart et al., 1997), a period that coincides with limited myelination (Jhaveri et al., 1992; Keirstead et al., 1992;
Ghooray and Martin, 1993; Kapfhammer and Schwab, 1994; Varga et al., 1995; MacLaren, 1996). These experiments suggest that failure to regenerate is due to growth-inhibitory molecules on myelin, or molecules that are strongly adhesive and which prevent axons from rapid growth. Thus, embryonic neurons would not express such ligands and are able to grow in the mature central nervous system (Wictorin et al., 1990; Davies et al., 1993; Li and Raisman, 1993; Li et al., 1995; Shewan et al., 1995; Stichel et al., 1995; Dusart et al., 1997).

Similar data on the failure of axons to reinnervate their targets in slice cultures were reported (Chen et al., 1995; Li et al., 1995; Dusart et al., 1997). These studies revealed an age-related failure of axon regeneration due to the intrinsic maturational changes of axons themselves rather than to the extrinsic changes of their environment. In our case, axons can grow for a long distance inside each side of the interface; however, they do not cross the lesion site. Some axons grew along the lesion site without crossing it, suggesting inhibitory cues within the lesion area. Moreover, when axons cross the lesion, they do not make it to the contralateral target field. Substrate experiments revealed a remaining outgrowth capacity of IC axons. Growing axons became more restricted to the nonneuronal perimeter with maturation, as discussed below.

The decrease of axonal outgrowth seems to be an age dependent rather than a structure-specific phenomenon. Coculturing slices of the SOC with the IC revealed a similar failure of axons to cross the injury. However, the number of crossing axons is higher in the IC-SOC cocultures than in the IC-IC ones, revealing a great capacity of axonal regeneration in the first case. Although it was not easy to delineate the exact origin of individual crossing axon (IC or SOC), it looks as though the majority of these axons come from the SOC area, especially the large caliber axons that may represent nonauditory neurons of the reticular formation, and these axons may have a different growth potential.

Substrate experiments were designed to address whether growth-inhibitory activity was due to the presence of inhibitory cues within the axonal environment. If given a favorable substrate, the axons may display a normal outgrowth (David and Aguayo, 1981; Li et al., 1997; Ramon-Cueto et al., 1998). The substrate experiments showed a decrease in the axonal growth depended on tissue age, as well as the growth media. The greatest amount of growth was found in P6 explants grown in N2-defined medium [Fig. $7(\mathrm{~A}, \mathrm{~B})]$. The least outgrowth onto the substrate was found in P12 explants grown in serum-containing 
medium. Serum-containing medium is known to enhance glial cell proliferation, and highly proliferative astrocytes are less supportive of neurite outgrowth (Banker and Kimberly, 1991; Wang et al., 1994). Therefore, the explant experiments suggest that the lesion site in P12 tissue may be populated by proliferating astrocytes that express growth-inhibiting molecules (McKeon et al., 1991; Carbonetto, 1991). This suggests that the astrocyte response to injury changes with age, since axons do grow across the lesion site in P6 explants of the inferior colliculus (Hafidi et al., 1995).

The appearance of MAG during development (Hafidi et al., 1995) correlates with the inhibitory growth of axons in this nucleus. Oligodendrocytes in older culture slices showed a more mature and reactive state, as revealed by the strong MAG immunostaining in comparison to P6 slices (Hafidi et al., 1995). MAG has been shown in vitro to have an inhibitory activity for neurite outgrowth (Mckerracher et al., 1994; Mukhopadhyay et al., 1994) and disruption of the gene for MAG improves axonal regrowth (Schafer et al., 1996).

In summary, the progressive decrease in the regenerative process may be essentially due to two phenomena. The first is the changing cues given by the environment (David and Aguayo, 1981), essentially owing to the maturation of glia. The second explanation is a decrease in the capacity of the axons themselves to grow (Fawcett, 1992; Chen et al., 1995; Li et al., 1995). The experiments using the substrate favors the latter proposition. Our experiments reconcile the two hypotheses in that there is both a decrease in the axonal growth in old IC tissue and an increase in the maturation of the glial environment.

This work was supported by NIH Grant DC00540.

\section{REFERENCES}

Agnew WF, McCreery DB, Yuen TG, Bullara LA. 1993. MK-801 protects against injury induced by electrical stimulation. Neuroscience 52:45-53.

Aguayo AJ, Bray GM, Rasminsky M, Zwimpfer T, Carter D, Vidal-Sanz M. 1990. Synaptic connections made by axons regenerating in the central nervous system of adult mammals. J Exp Biol 153:199-224.

Bandtlow C, Zachleder T, Schwab ME. 1990. Oligodendrocytes arrest neurite growth by contact inhibition. J Neurosci 10:3837-3848.

Banker G, Kimberly G. 1991. Culturing nerve cells. Cambridge, MA: MIT Press.

Berry M, Carlile J, Hunter A. 1996. Peripheral nerve explants grafted into the vitreous body of the eye promote the regeneration of retinal ganglion cell axons severed in the optic nerve. J Neurocytol 25:147-170.

Bregman BS, Goldberger ME. 1983. Infant lesion effect. III. Anatomical correlates of sparing an recovery of function after spinal cord damage in newborn and adult cats. Brain Res 285:137-154.

Brorson JR, Manzolillo PA, Miller RJ. 1994. Ca2+ entry via AMPA/KA receptors and excitotoxicity in cultured cerebellar Purkinje cells. J Neurosci 14:187-197.

Ramón y Cajal S. 1928. Regeneration and degeneration of the nervous system. (Reprint) (May RM, Trans.). New York: Hafner, 1959.

Carbonetto S. 1991. Facilitatory and inhibitory effects of glial cells and extracellular matrix in axonal regeneration. Curr Opin Neurobiol 1:407-413.

Chen DF, Jhaveri S, Schneider GE. 1995. Intrinsic changes in developing retinal neurons result in regenerative failure of their axons. Proc Natl Acad Sci USA 92:72877291.

Choi DW, Hartley DM. 1993. Calcium and glutamateinduced cortical neuronal death. In: Waxman SG, editor. Molecular and cellular approaches to treatment of neurological disease. New York: Raven Press. p 23-34.

Choi DW, Koh JY, Peters S. 1988. Pharmacology of glutamate neurotoxicity in cortical cell culture: attenuation by NMDA antagonists. J Neurosci 8:185-196.

Clarke PGH. 1990. Developmental cell death: morphological diversity and multiple mechanisms. Anat Embryol 181:195-213.

David S, Aguayo AJ. 1981. Axonal elongation into peripheral nervous system "bridges" after central nervous system injury in adult rats. Science 214:931-933.

Davies SJA, Field PM, Raisman G. 1993. Long fibre growth by axons of embryonic mouse hippocampal neurons microtransplanted into the adult rat fimbria. Eur J Neurosci 5:95-106.

Davies SJA, Fitch TM, Memberg SP, Hall AK, Raissman G, Silver J. 1997. Regeneration of adult axons in white matter tracts of the central nervous system. Nature 390: 680-683.

Didier M, Héaulme M, Gonalons N, Soubrié P, Boackert J, Pin JP. 1993. $35 \mathrm{mM} \mathrm{K}+$ stimulated ${ }^{45} \mathrm{Ca}^{2+}$ uptake in cerebellar granule cell cultures mainly results from NMDA receptor activation. Eur J Pharmacol 244:57-65.

Dusart I, Airaksinen MS, Sotelo C. 1997. Purkinje cell survival and axonal regeneration are age dependent: an in vitro study. J Neurosci 17:3710-3726.

Ellren K, Lehmann A. 1989. Calcium dependency of $N$ methyl-D-aspartate toxicity in slices from the immature rat hippocampus. Neuroscience 132:371-379.

Fawcett JW, Housden E, Smith-Thomas L, Meyer RL. 1989. The growth of axons in three-dimensional astrocyte culture. Dev Biol 135:449-458.

Fawcett JW. 1992. Intrinsic neuronal determinants of regeneration. Trends Neurosci 15:5-8.

Franklin JL, Sanz-Rodriguez C, Juhasz A, Deckwerth TL, Johnson EM. 1995. Chronic depolarization prevents programmed death of sympathetic neurons in vitro but does 
not support growth: requirement for $\mathrm{Ca}$ influx but not Trk activation. J Neurosci 15:643-664.

Ghoory GT, Martin GF. 1993. The development of myelin in the spinal cord of the North American opossum and its possible role in loss of rubrospinal plasticity: a study using myelin basic protein and galactocerebrocide immuno-histochemistry. Dev Brain Res 72:67-74.

Hafidi A, Moore T, Sanes DH. 1996. Regional distribution of neurotrophin receptors in the developing auditory brainstem. J Comp Neurol 367:454-464.

Hafidi A, Sanes DH, Hillman DE, Kedeshian P. 1994. Structural and molecular distribution of oligodendrocytes and astrocytes along the tonotopic axis of the gerbil lateral superior olive. Neuroscience 63:503-519.

Hafidi A, Sanes DH, Hillman DE. 1995. Regeneration of the auditory midbrain intercommissural projection in organotypic culture. J Neurosci 15:1298-1307.

Hasan SJ, Keirsteid HS, Muir GD, Steeves JD. 1993. Axonal regeneration contributes to repair of injured brainstem-spinal neurons in embryonic chick. J Neurosci 13: 492-507.

Hori N, Ffrench-Mullen JMH, Carpenter DO. 1985. Kainic acid responses and toxicity show pronounced $\mathrm{Ca}^{2+}$ dependence. Brain Res 358:380-384.

Jhaveri S, Erzurumlu RS, Friedman B, Schneider GE. 1992. Oligodendrocytes and myelin formation along the optic tract of the developing hamster: an immunohistochemical study using the Rip antibody. Glia 6:138-148.

Kalil K, Reh T. 1982. A light and electron microscopic study of regrowing pyramidale tract fibers. J Comp Neurol 211:265-275.

Kapfhammer JP, Schwab ME. 1994. Inverse patterns of myelination and GAP43 expression in the adult CNS: neurite growth inhibitors as regulators of neuronal plasticity? J Comp Neurol 340:194-206.

Keirstead HS, Hasan SJ, Muir GD, Steeves JD. 1992. Suppression of the onset of myelination extends the permissive period for functional repair of embryonic spinal cord. Proc Natl Acad Sci USA 89:1164-1168.

Kingsbury A, Balazs R. 1987. Effect of calcium agonists and antagonists on cerebellar granule cells. Eur J Pharmacol 140:275-283.

Koh JY, Gwag BJ, Lobner D, Choi DW. 1995. Potentiated necrosis of cultured cortical neurons by neurotrophins. Science 268:573-575.

Li D, Field PM, Raisman G. 1995. Failure of axon regeneration in postnatal rat entorhino-hippocampal slice coculture is due to maturation of the axon, not that of the pathway or target. Eur J Neurosci 7:1164-1171.

Li D, Field PM, Raisman G. 1997. Repair of adult rat corticospinal tract by transplants of olfactory ensheathing cells. Science 277:2000-2002.

Li D, Raisman G. 1993. Long axon growth from embryonic neurons transplanted into myelinated tracts of the adult rat spinal cord. Brain Res 629:115-127.

Lo Y-J, Rao SC, Sanes DH. 1998. Modulation of calcium by inhibitory systems in the developing auditory system. Neuroscience 83:1075-1084.
Lohmann C, Llic V, Friauf E. 1997. Development of a topographically organized auditory network in slice culture is calcium dependent. J Neurobiol 34:97-112.

Lu YM, Yin HZ, Chiang J, Weiss JH. 1996. Ca2+-permeable AMPA/Kainate and NMDA channels: high rate of $\mathrm{Ca} 2+$ influx underlies potent induction of injury. J Neurosci 16:5457-5465.

MacDermott AB, Mayer ML, Westbrook GL, Smith SJ, Barker JL. 1986. NMDA-receptor activation increases cytoplasmic calcium concentration in cultured spinal cord neurones. Nature 321:519-522.

Mackerracher L, David S, Jackson DL, Kottis V, Dunn RJ, Braun PE. 1994. Identification of myelin-associated glycoprotein as a major myelin derived inhibitor of neurite growth. Neuron 13:805-811.

MacLaren RE. 1996. Expression of myelin proteins in the opossum optic nerve: late appearance of inhibitors implicates an earlier non-myelin factor in preventing ganglion cell regeneration. J Comp Neurol 372:27-36.

MacLaren RE, Taylor JSH. 1995. A critical period for axon regrowth through a lesion in the developing mammalian retina. Eur J Neurosci 10:2111-2118.

Mattson MP, Cheng B, Smith-Swintosky VL. 1993. Mechanisms of neurotrophic factor protection against calcium and free radical-mediated excitotoxic injury: implications for treating neurodegenerative disorders. Exp Neurol 124: 89-95.

McKeon RJ, Schreiber RC, Rudge JS, Silver J. 1991. Reduction of neurite outgrowth in a model of astroglial scarring following CNS injury is correlate with the expression of inhibitory molecules on reactive astrocytes. J Neurosci 11:3398-3411.

Michaels RL, Rothman SM. 1990. Glutamate cytotoxicity in vitro: antagonist pharmacology and intracellular calcium concentration. J Neurosci 10:283-292.

Moore DR, Kotak VC, Sanes DH. 1998. Commissural and lemniscal synaptic input to the gerbil inferior colliculus. J Neurophysiol 80:2229-2236.

Mukhopadhyay G, Doherty P, Walsh FS, Crocker PR, Filbin MT. 1994. A novel role for myelin-associated glycoprotein as an inhibitor of axonal regeneration. Neuron 13:757-767.

Portera-Cailliau C, Hedreen JC, Price DL, Koliatsos VE. 1995. Evidence of apoptotic cell death in Huntington disease and excitotoxic animal models. J Neurosci 15: 3775-3787.

Pozzo Miller LDP, Mahanty NK, Connor JA, Landis DMD. 1994. Spontaneous pyramidal cell death in organotypic slice cultures from rat hippocampus is prevented by glutamate receptor antagonists. Neuroscience 63:471-487.

Ramon-Cueto A, Plant GW, Avila J, Bunge MB. 1998. Long-distance axonal regeneration in the transected adult rat spinal cord is promoted by olfactory ensheathing glia transplants. J Neurosci 18:3803-3815.

Reier PJ, Stensaas LJ, Guth L. 1983. The astrocytic scar as an impediment to regeneration in the central nervous system. In: Kao CC, Bunge RP, Reier PJ, editors. Spinal cord reconstruction. New York: Raven. p 163-195. 
Richardson PM, McGuiness UM, Aguayo AJ. 1980. Axons from CNS neurones regenerate into PNS grafts. Nature 254:264-265.

Rothman SM, Thurston JH, Hauhart RE. 1987. Delayed neurotoxicity of excitatory amino acids in vitro. Neuroscience 22:471-480.

Rudge JS, Silver J. 1990. Inhibition of neurite outgrowth on astroglial scars in vitro. J Neurosci 10:3594-3603.

Sanes DH, Hafidi A. 1996. Glycine receptor blockade alters dendrite size in organotypic culture. J Neurobiol 31:503511.

Sanes DH, Malone BL, Semple MN. 1998. Modulation of binaural level stimuli in gerbil inferior colliculus: role of synaptic inhibition. J Neurosci 18:794-803.

Sanes DH, Wooten GF. 1987. Development of glycine receptor distribution in the lateral superior olive of the gerbil. J Neurosci 7:3803-3811.

Schafer M, Fruttiger M, Montag D, Schachner M, Martini R. 1996. Disruption of the gene for the myelin-associated glycoprotein improves axonal regrowth along myelin in C57BL/Wld mice. Neuron 16:1107-113.

Schmidt MF, Kater SB. 1993. Fibroblast growth factors, depolarization and substratum interact in a combinatorial way to promote neuronal survival. Dev Biol 158:228237.

Shen RY, Altar A, Chiodo LM. 1994. Brain derived neurotrophic factor increases the electrical activity of pars compacta dopamine neurons in vivo. Proc Natl Acad Sci USA 91:8920-8924.

Shewan D, Berry M, Cohen J. 1995. Extensive regeneration in vitro by early embryonic neurons on immature and adult tissue. J Neurosci 15:2057-2062.

Shimizu I, Oppenheim RW, O’Brien M, Schneiderman A. 1990. Anatomical and functional recovery following spinal cord transection in the chick embryo. J Neurobiol 21:918-937.

Solum D, Hughes D, Major MS, Parks TN. 1997. Prevention of normally occurring and deafferentation-induced neuronal death in chick brainstem auditory neurons by periodic blockade of AMPA/kainate receptors. J Neurosci 17:4744-4751.

Sperk G, Lassmann H, Baran H, Kish SJ, Seitelberger F, Hornykiewicz O. 1983. Kainic acid induced seizures: neurochemical and histopathological changes. Neuroscience 10:1301-1315.

Stichel CC, Wunderlich G, Schwab ME, Muller HW. 1995. Clearance of myelin constituents and axonal sprouting in the transected postcommissural fornix of the adult rat. Eur J Neurosci 7:401-411.
Stoppini L, Buchs LA, Muller D. 1991. A simple method for organotypic cultures of nervous tissue. J Neurosci Methods 37:173-182.

Thornton S, Semple MN, Sanes DH. (1999). Development of auditory motion processing in the gerbil inferior colliculus. Eur J Neurosci 11:1414-1420.

Tymianski M, Charlton MP, Carlen PL, Tator CH. 1994. Properties of neuroprotective cell-permeant $\mathrm{Ca}^{++}$chelators: effects on $\left[\mathrm{Ca}^{++}\right] \mathrm{i}$ and glutamate neurotoxicity in vitro. J Neurophysiol 72:1973-1992.

Vale C, Sanes DH. 1999. The effects of bilateral cochlear ablation on synaptic inhibition in the gerbil inferior colliculus. Assoc Res Otolaryngol Abstr 22.

Van-Lookeren Campagne M, Lucassen PJ, Vermeulen JP, Balazs R. 1995. NMDA and kainate induce internuclear DNA cleavage associate with both apoptotic and necrotic cell death in the neonatal rat brain. Eur $\mathrm{J}$ Neurosci 7:1627-1640.

Varga ZM, Bandtlow CE, Erulkar SD, Schwab ME, Nicholls JG. 1995. The critical period for repair of CNS of neonatal opossum (Monodelphis domestica) in culture: correlation with the development of glial cells, myelin and growth-inhibitory molecules. Eur J Neurosci 7:2119-2129.

Vornov JJ, Tasker RC, Park J. 1995. Neurotoxicity of acute glutamate transport blockade depends on coactivation of both NMDA and AMPA/kainate receptors in organotypic hippocampal cultures. Exp Neurol 133:7-17.

Wang LC, Baird DH, Hatten ME, Mason CA. 1994. Astroglial differentiation is required for support of neurite outgrowth. J Neurosci 14:3195-3207.

Wang XM, Terman JR, Martin GF. 1996. Evidence for growth of supraspinal axons through the lesion after transection of the thoracic spinal cord in the developing opossum Didelphis virginiana. J Comp Neurol 371:104115.

Watanabe M, Inoue Y, Sakimura K, Mishina N. 1994. Distinct distributions of five $N$-methyl-D-aspartate receptor channel subunit mRNAs in the forebrain. J Comp Neurol 338:377-390.

Wictorin K, Brundin P, Gustavii B, Lindvall O, Bjorklund A. 1990. Reformation of long axon pathways in adult rat central nervous system by human forebrain neuroblasts. Nature 347:556-558.

Wyllie AH, Kerr JFR, Currie AR. 1980. Cell death: the significance of apoptosis. Int Rev Cytol 68:251-306.

Xu XM, Martin GF. 1989. Developmental plasticity of the rubrospinal tract in the north American opossum. J Comp Neurol 279:368-381. 